\title{
Single vs. extended antibiotic for prevention of surgical infection in emergent cesarean delivery
}

\begin{abstract}
Objective: Emergency Cesarean Section (ECS) improves maternal and neonatal outcomes but can be associated with complications including Surgical Site Infections (SSI). Prophylactic antibiotics reduce SSIs but inappropriate use increases antibiotic resistance. The study aim was to assess single versus multiple-dose antibiotic prophylaxis for SSI prevention in a limited-resource setting.
\end{abstract}

Methods: All patients undergoing ECS in a rural district hospital were assessed for eligibility from 2015 to 2016. Participants were randomized, Group A received one dose of $2 \mathrm{~g}$ Ampicillin 15 to 60 minutes before skin incision and Group B received $2 \mathrm{~g}$ prior to skin incision with additional $1 \mathrm{~g}$ every 8 hours over 72 hours. Participants were followed for 30 days, Demographic and clinical data were collected by chart review and patient phone interviews were performed on Days 3, 7, 15 and 30 .

Results: Three hundred and one participants were analyzed (147 in Group A; 154 in Group B). There were 8 surgical site infections in Group A and 4 in Group B ( $\mathrm{p}=0.089)$. The overall prevalence of surgical site infections was lower than expected (4.00\%). Most of SSIs cases were diagnosed on day 7 (66.6\% of all cases of SSI) and only $22.2 \%$ of cases were diagnosed at discharge (day 3). Only 1 additional case was diagnosed at day 15 .

Conclusion: Compliance to infection control measures can reduce the rate of SSIs. There was no significant difference between the use of a single vs multiple doses of prophylactic antibiotics in our study but the SSI rate was low.
Volume 7 Issue 2 - 202I

\author{
David Nitrushwa, ${ }^{1,5}$ Rahel Ghebre, ${ }^{1,2,6}$ Marie \\ A Unyuzimana, ${ }^{3}$ Urania Magriples, ${ }^{1,2}$ Maria \\ Small, ${ }^{1,4}$ Stephen Rulisa ${ }^{1,5}$ \\ 'Department of Obstetrics and Gynecology, School of Medicine \\ and Pharmacy, University of Rwanda, Rwanda \\ ${ }^{2}$ Department of Obstetrics, Gynecology, and Reproductive \\ Sciences, Yale School of Medicine, USA \\ ${ }^{3}$ Medical department, Project San Francisco/Center for Family \\ Health Research, Rwanda \\ ${ }^{4}$ Department of Obstetrics and Gynecology, Duke Medical \\ Center, USA \\ ${ }^{5}$ Department of Obstetrics and Gynecology, University Teaching \\ Hospital of Kigali(CHUK), Rwanda \\ ${ }^{6}$ Department of Obstetrics and Gynecology, University of \\ Minnesota Medical School, Rwanda
}

\section{Correspondence: David Ntirushwa, Consultant Obstetrician and Gynecologist, Rwanda, Tel 250788744660,} Email dagrain002@gmail.com

Received: April 02, 202I | Published: April 30, 202 |

Keywords: antibiotic, post-surgery, prevention, prophylactic antibiotics

Abbreviations: SSI, surgical site infection; THRiVE, The training health researchers into vocation excellence in East Africa; WHO, World health organization; C/S, cesarean section; HIV, human immunodeficiency virus; $\mathrm{CDC}$, center for disease control and prevention; $\mathrm{MD}$, multiple doses; $\mathrm{SD}$, single dose; $\mathrm{HRH}$, human resource for health; PI, principle investigator

\section{Introduction}

Cesarean section is a lifesaving intervention which contributes to improved maternal and neonatal outcome when performed appropriately. The World Health Organization (WHO) has considered the optimal rate of cesarean rate to be between 10 and $15 \%$ but the numbers have been increasing in both developed and developing countries. ${ }^{1}$ Unfortunately, as with any surgical intervention, cesarean section can lead to undesired complications including post cesarean infections. Surgical site infection (SSI), which involves the skin and uterine incisions, is frequent and can cause short and long term adverse outcomes. SSI following cesarean section is a major cause of morbidity and mortality, increasing both the duration of hospitalization and hospital costs as well as overall patient dissatisfaction. ${ }^{2}$

The increase in cesarean sections rates and the associated postsurgery complication mainly infections has attracted the interest of medical researchers to examine and determine the best way antimicrobial prophylaxis can be used efficiently to prevent SSIs. Three main areas around the use of antibiotics have been attracting interest for evidence and include; the timing of administrating antibiotics, types of antibiotics and duration of antibiotics; single-dose versus multiple doses.
Currently robust evidence support the role of antibiotics in preventing post-cesarean infections and the use of antibiotics prior to surgical incision is considered to be effective in reducing cases of SSI. ${ }^{3}$ As proven in a recent study done by Zuarez-Easton $\mathrm{S}$ et al , compared to placebo, the use of prophylactic antibiotics during cesarean section significantly reduce the incidence of wound infection (RR: 0.40, 95\% CI: 0.35-0.46), endometritis (RR: 0.38, 95\% CI: $0.34-0.42$ ), and maternal serious infectious complications (RR: 0.31 , $95 \%$ CI: $0.20-0.49){ }^{4}$

Even though, antibiotics are widely used globally to prevent morbidity and mortality from maternal infections post cesarean section, the misuse of antibiotics for obstetrics conditions and procedures that are thought to carry the risk of maternal infections is a common practice that can potentially lead to other consequences including resistance to antibiotics. The 2015 WHO recommendations for the prevention and treatment of maternal puerperium infections recommend a single dose of first-generation cephalosporin or penicillin for prevention of post cesarean infections but with weak evidence. ${ }^{5}$ Considering the timing of giving prophylactic antibiotics, It was previously feared that administrating antibio-prophylaxis before skin incision could affect the baby in utero, however, according to several studies, there is strong evidence that prophylactic antibiotic for cesarean delivery given before skin incision rather than after cord clamping decreases the incidence of postpartum endometritis and total infectious morbidities without affecting neonatal outcomes. ${ }^{6}$ Another important aspect of prophylactic antibiotic for cesarean section is related to the type of antibiotics to use, the choice has to be made based on the type of common organisms likely to cause 
postpartum infections especially SSIs and endometritis to ensure a targeted coverage. The antibiotics must be present not only in the plasma but also in the tissues during the surgical procedure, and in most cases, very short prophylaxis, usually using a single bolus is convenient. ${ }^{7}$ In 2004 E. Ahmed et al. found no difference between a single dose of ceftriaxone versus Ampicillin/Cloxacillin given at the induction of anesthesia. ${ }^{8}$ A Cochrane systematic review done by Dahlke JD et al ,recommended that a single dose of ampicillin or firstgeneration cephalosporins, such as cefazolin, should be administered in all women undergoing cesarean delivery and given 15 to 60 minutes before skin incision. ${ }^{9}$ According to various research findings, evidence supports the use of pre-skin incision broad-spectrum antibiotics ${ }^{10}$ and there is insufficient evidence to determine whether there is a difference between the use single dose versus multiple-dose regimens . In 1988 a multicenter comparative study done by Galask RP et al in the USA compared a single dose of cefotetan versus multiple-dose cefoxitin as prophylaxis in patients undergoing cesarean section and no difference in cases of endometritis or surgical site infection was seen. ${ }^{11}$ Despite all the evidence about the use of a single dose of antibiotics, resistance to change is common in many hospitals; for example, in a teaching hospital, southern Taiwan, dissemination of evidence was done and clinicians were informed about single-dose prescription as evidencebased best practice and follow-up evaluations were made to see how the information has helped clinicians change their practices. The rate of single-dose prescription only increased from $14.2 \%$ to $22.4 \%$ and the study concluded that knowledge of evidence does not improve practice uniformly; more other interventions were encouraged to improve practice. ${ }^{12}$

Even though several studies performed in high-income countries still recommend the use of single dose prophylaxis, more cautions and hesitancy are taken in low resource settings. Various aspects including lack of sufficient infrastructures in operating rooms, lack of optimal asepsis, absence of clear antiseptic rules, reuse of disposable materials, challenges in postoperative follow up especially after discharge from the hospital and patients' level of education to maintain their hygiene, dictate the use of extended antibiotic prophylaxis after emergency cesarean section and even electives cesarean section by many health professionals in low resource settings. There is also insufficient data on the effectiveness of antibiotics, further limiting an evidencebased choice of antibiotics and biasing providers towards the use of antibiotics with a broader range.

As a consequence, we lack evidence-based standardized protocols to guide the duration of antimicrobial prophylaxis after emergency cesarean section in low-resource settings. Considering the consequences of inappropriate use of antibiotics and lack of evidencebased consensus about a clear protocol in low resources maternity settings, studies comparing a single-dose of antibiotics to multiple doses in uncomplicated emergency cesarean sections are necessary.

In 2012 at Bugando Medical Center in Tanzania, a study by Lyimo FM et al compared a single-dose of Gentamycin and Metronidazole 30 to 60 minute before skin incision versus extended use of the same antibiotics for 24 hours and found no difference in outcome and the use of single intravenous Gentamycin and metronidazole was found superior to an extended dose of the same antibiotics. ${ }^{13}$ The extended systematic use of antibiotics to patients with emergency cesarean section without obvious risk factors have some negative impacts on patient care; it leads to longer hospital stay, prolonged use of intravenous medications, increased cost for patients and hospitals, increase in the risk of acquiring nosocomial infections and increased risk of developing resistance to commonly used antibiotics. Emin et al studied the incidence and risk factors of SSI in developing countries and concluded that prolonged antimicrobial prophylaxis and broad spectrum antibiotics may be associated with the emergence of resistant bacterial strains. ${ }^{14}$ Recently some studies carried out in a tertiary hospitals in Rwanda have found a high prevalence of antimicrobial resistance to commonly used antibiotics; One of those studies was done by Ntirenganya $\mathrm{C}$ et al and found among culture isolates E.Coli and Klebsiella pathogens to be the most common and were resistant to at least one of the third-generation cephalosporins, other pathogens also had resistance to other commonly used antibiotics. ${ }^{15}$ Another study done by Muvunyi CM et al in Rwanda found out that the antibiotics commonly used in Rwanda for the treatment of UTI are no longer effective due to increased resistance of the most common pathogen to common antibiotics. ${ }^{16}$ Interventions aiming at reducing the risk of resistance and leading to the correct use of antibiotics would be helpful in Rwanda and in other countries where they are no clear protocols. There is no data in Rwanda about the prevalence of surgical site infections post caesarean section. There are limited studies performed in East Africa. A study done in Tanzania at Bugando Medical Center revealed an incidence of $6.4 \%$ among women who received prolonged antibiotic prophylaxis during emergency cesarean section while a study performed in Nigeria noted a prevalence of $9.1 \%$ with single-dose antibiotics. ${ }^{17}$

In this study, we aim to test if single-dose antimicrobial prophylaxis is as effective as extended doses of antibiotics for prevention of post caesarean section surgical site infections or if the use of extended antibiotics is justified in high risk poor resource settings. We also sought to determine the baseline incidence of SSI among women receiving antimicrobial prophylaxis after an emergency cesarean section.

\section{Methods}

A prospective parallel randomized clinical trial with 1:1 allocation ratio was performed. Sample size calculation was calculated based on previous estimates on SSI in the literature. Using the Blackwelder formula for equivalence RCTs, where we hypothesized a difference of less than $5 \%$ between the two treatments to represent the equivalence. We have used the SSI prevalence found in the previously cited study of $6.4 \%$ among women receiving multiple doses of antibio-prophylaxis and we used an incidence of $10 \%$ for single dose therapy based on the previously cited Nigerian study which was $9.1 \%{ }^{17}$

The Blackwelder formula used is: $\left(\mathrm{Z}_{0.95}+\mathrm{Z}_{0.80}\right)^{2}[\mathrm{Ps}(1-\mathrm{Ps})+\mathrm{Pn}(1-$ Pn)] / (Ps-Pn-D) $)^{2}$

Ps: 6,4\% Ps: Estimated Response among women receiving the standard treatment (Multiple doses)

Pn: 10 \%; Pn: Estimated Response rate among women receiving the new treatment (Single dose)

\section{D: $5 \%$ : Hypothesized difference for equivalence}

Using the above prevalence, the sample size is 125 for each group and 250 for both groups. We assumed that $10 \%$ of patients may be lost to follow up or have incomplete documentation therefore the total sample size was calculated at 275 patients. All the calculation assume alpha is $=0,05$ and beta is $=0,2$ (power $80 \%$ ). A statistician who was not involved in enrolling patients used computer-generated random numbers with block randomization. The size of the blocks was only known by the statistician and blinded to those who enroll participants. All the random numbers were sealed in opaque envelopes and kept in a sized box. Each participant picked the next envelope before cesarean section and the envelope was opened at the end of surgery. Each envelope contained a random number corresponding to either a single dose or multiple doses depending on what the computer had generated. 
Three hundred one women have been recruited, enrolled and analyzed in the study from $1^{\text {st }}$ July 2015 to June 2016. With a 1:1 allocation ratio, 147 women received a single dose of antibiotics; 2 gms of Ampicillin 15-60 minutes before skin incision and 154 women received 2 gms 15-60 minutes prior to skin incision and continued 1 gm every 8 hours for 3 days. Our study was conducted in Ruhengeri Hospital, a large rural hospital in Rwanda. It has a maternity department where approximately 5000 women deliver annually with around 1500 deliveries being cesarean sections.

Eligibility criteria included all women admitted in maternity at Ruhengeri Hospital during the study period and had agreed to be enrolled in the study after a written informed consent was obtained. Women who had signs of ongoing infections such as chorioamnionitis, fever during labor, premature rupture of membranes for more than 18 hours and those who have had failed instrumental delivery at complete dilatation were excluded. All study participants received 2 grams of Ampicillin in labor ward before entering the operating room to meet the required 15-60 minutes before skin incision. While the participants were in the operating room, the provider was given the sealed opaque envelope containing a number that designated whether the patients were in the category of single dose which was already given in labor ward or whether the patient was a candidate for extended use of antibiotics for 3 days more. The envelope was opened only once the cesarean section is over to ensure that the surgeon is blinded to the regimen during the surgery.

All participants were educated about the signs of surgical site infections which were defined as fever, tenderness and swelling of the wound or loss of continuity with pus discharge. Participants were evaluated for any sign of SSI on the day of discharge (day 3), then followed by phone interviews on day 7,15 and day 30. All patients were requested to come back to the same hospital if any problem during the follow up was to occur. Secondary outcomes included endometritis and any other type of postpartum infection. Participants were also educated about signs of endometritis, which were defined as fever, tender abdomen and full smelling discharge. Participants were requested to consult if any of the above symptoms were observed during the follow up period.

\section{Statistical analysis}

A questionnaire containing the social demographic and clinical characteristics was used at the time of enrollment and during follow up. Data on social demographic and clinical characteristics were extracted in the file at the time of enrollment and discharge on day 3. The source of data during follow up on Day 7,15 and 30 was obtained through an interview on phone and all patients were requested to come back to Ruhengeri Hospital whenever they have any concerns; Patients were also allowed and given the contact of the PI whom they can call whenever they have any issue. Note that patients were educated about signs of SSI and other infections and were interviewed about those signs during the follow-up. The follow up was made possible by using the phone call directly to the patient or the husband in case of the participants had no phone or community health worker in charge of the patient.

Data were entered through epi data and then extracted into Stata 13 for analysis. Descriptive statistics were used to test whether the population in both groups were similar and the incidence of SSI in both groups was calculated on day 3 at discharge, on day 7, day 15 and day 30. The overall incidence of SSI in each group was used to calculate the difference and $\mathrm{P}$ value considered for the significance of the observed difference. The equivalence margin above 5\% was initially set as the required difference to confirm the difference in both groups within the $95 \%$ confidence interval.

\section{Results}

\section{Baseline characteristics in our study population}

A total of 618 patients were assessed for eligibility. Among them, 20 participants declined to participate in the study and 50 were excluded because they were not meeting the inclusion criteria or did not receive timely antibiotics. The rest of excluded participants (247) were secondary to the provider not willing to participate in the study blaming lack of time for enrolment and randomization due to work overload . A total of 301 women were enrolled, randomized and analyzed; 154 in the group of multiple- dose and 147 in the group of a single-dose, basic social demographic and clinical characteristics were similar in both groups as shown in Table 1.

Table I Social demographic and clinical characteristics in the two groups

\begin{tabular}{|c|c|c|c|c|}
\hline & & SD & M D & $P$ value \\
\hline \multirow[t]{4}{*}{ Age } & Categories & & & 0.144 \\
\hline & Less than 20 & $6(4.5 \%)$ & $6(4.2 \%)$ & \\
\hline & $20-35 y r a$ & $119(90.2 \%)$ & $120(84.5 \%)$ & \\
\hline & 36 and above & $7(5.3 \%)$ & $16(11.3 \%)$ & \\
\hline \multirow[t]{5}{*}{ Level of education } & & & & 0.028 \\
\hline & No formal education & $32(22.4 \%)$ & $24(\mid 5.8 \%)$ & \\
\hline & Primary & $83(58 \%)$ & $82(53.9 \%)$ & \\
\hline & Secondary & $22(15.4 \%)$ & $36(23.7 \%)$ & \\
\hline & University & $6(4.2 \%)$ & $10(6.6 \%)$ & \\
\hline \multirow[t]{3}{*}{ History of SSI } & & & & 0.749 \\
\hline & Yes & $\mathrm{I}(.89 \%)$ & $\mathrm{I}(.89 \%)$ & \\
\hline & No & II I(99.10\%) & III(99.10\%) & \\
\hline
\end{tabular}




\begin{tabular}{|c|c|c|c|}
\hline & SD & M D & $P$ value \\
\hline \multicolumn{3}{|l|}{ HIV status } & 0.088 \\
\hline Negative & $66(45.5 \%)$ & $83(54.5 \%)$ & \\
\hline Unknown & $-49.10 \%$ & $50(44.64 \%)$ & \\
\hline \multicolumn{3}{|l|}{ Diabetes } & 0.728 \\
\hline Negative & $18(12.4 \%)$ & $17(11.1 \%)$ & \\
\hline Positive & $0(0 \%)$ & $0(1.3 \%)$ & \\
\hline Unknown & $127(87.6 \%)$ & | 36(88.9\%) & \\
\hline \multicolumn{3}{|l|}{ Indication for CS } & 0.166 \\
\hline Failure to progress & $27(19.0 \%)$ & $23(23.2 \%)$ & \\
\hline Fetal distress & $33(23.2 \%)$ & $31(20.4 \%)$ & \\
\hline Breech & $14(9.9 \%)$ & $12(7.9 \%)$ & \\
\hline Others & $68(47.9 \%)$ & $86(56.6 \%)$ & \\
\hline \multicolumn{3}{|l|}{ CS intervention duration } & 0.446 \\
\hline $0-2$ hours & $107(95.53 \%)$ & $110(98.21 \%)$ & \\
\hline More than 2 hours & $5(4.46 \%)$ & $2(1.78 \%)$ & \\
\hline \multicolumn{3}{|l|}{ Time spent in labor before CS } & 0.77 \\
\hline $0-1$ hours & $62(43.4 \%)$ & $74(49.0 \%)$ & \\
\hline I-5 hours & $56(39.2 \%)$ & $46(30.5 \%)$ & \\
\hline More than 5 hours & $25(17.5 \%)$ & $3 \mathrm{I}(20.5 \%)$ & \\
\hline
\end{tabular}

Below is the flow diagram indicating the recruitment, enrolment and follow up process

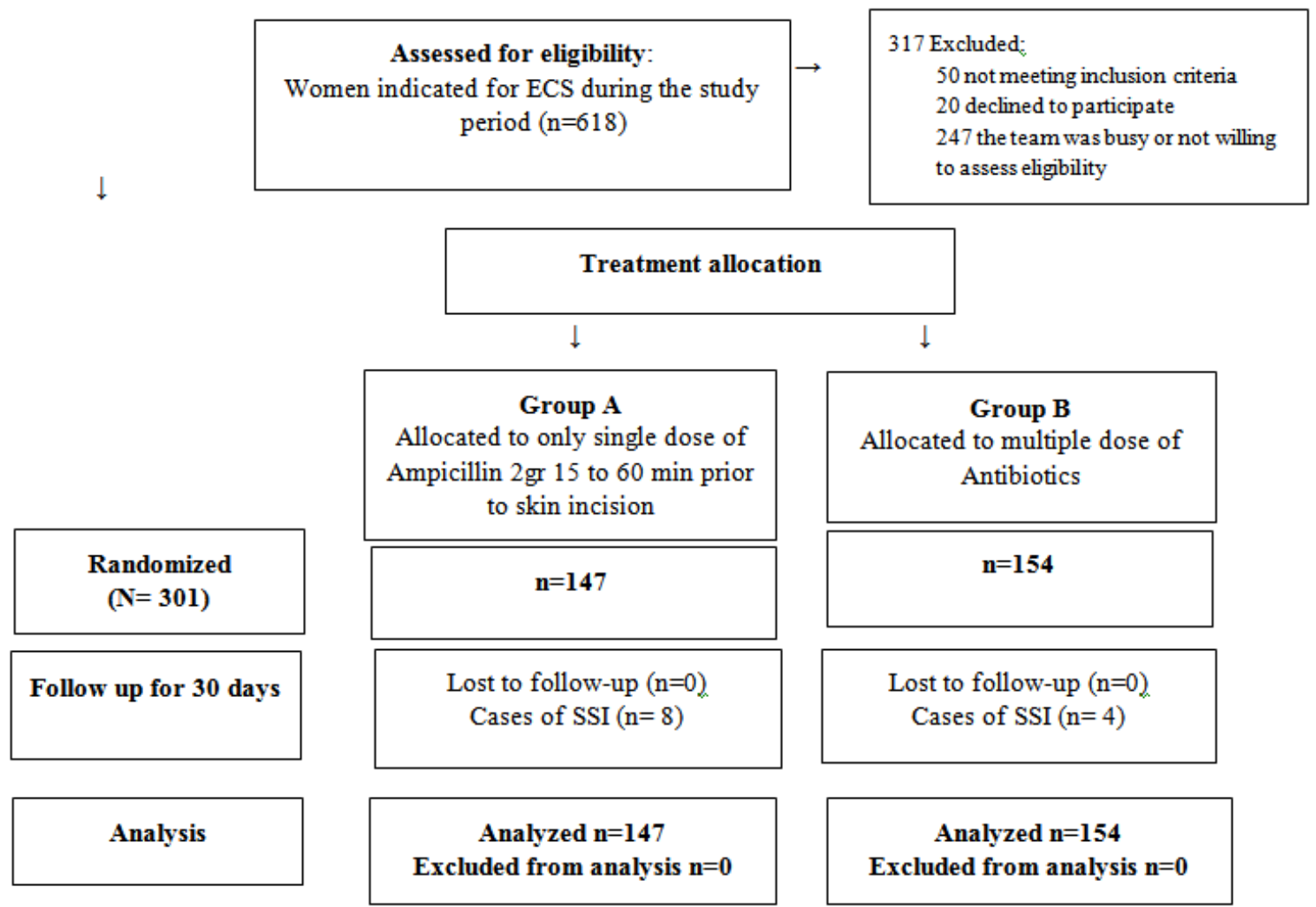

Citation: Nitrushwa D, Ghebre R, Unyuzimana MA, et al. Single vs. extended antibiotic for prevention of surgical infection in emergent cesarean delivery. Int J Pregn \& Chi Birth. 202I;7(2):5I-56. DOI: 10.15406/ipcb.2021.07.00228 


\section{Incidence of surgical site infection in both groups}

Among women who received a single dose of ampicillin, 8 women (5.5\%) developed SSI during the 30 days of follow-up, whereas 4 women $(2.6 \%)$ developed SSI among those who received multiple doses of antibiotics. The overall prevalence of SSI in our population was $4.00 \%$, which was surprisingly low compared to the baseline assumptions, which were calculated based on the literature available from other African countries. Most of the new cases of SSI were diagnosed on day 7. Only 2 cases of SSI were diagnosed at the time of discharge while no cases were newly diagnosed on day 30 (Tables 2\&3).

Table 2 Incidence of SSI in single versus multiple dose users within a period on 30 days

\begin{tabular}{lllll}
\hline & Regimen & & & \\
\hline & Single dose & Multiple dose & Total & P value \\
\hline None Surgical site wound infection & $138(94.5 \%)$ & $150(97.4 \%)$ & $288(96 \%)$ & 0.204 \\
Surgical site wound infection & $8(5.5 \%)$ & $4(2.6 \%)$ & 12(4.0\%) Overall incidence \\
\hline
\end{tabular}

Table 3 Incidence of SSI on day 3, 7, I5 and 30 in both groups

\begin{tabular}{llll}
\hline $\begin{array}{l}\text { Status of the } \\
\text { patient }\end{array}$ & Regimen & & \\
\hline & Single dose & Multiple dose & P Value \\
\hline Day 3 & & & 0.224 \\
& I44(97.9\%) & $154(100 \%)$ & \\
& $3(2.04 \%)$ & $0(0.0 \%)$ & 0.2 \\
Day7 & & & \\
& & & \\
& & & \\
& & & \\
& & & $0.083(2.70 \%)$ \\
\hline
\end{tabular}

\section{Others factors thought to have an impact on the risk of post cesarean surgical site infections}

Logistic regression was performed to analyze other cofactors that might have an impact on our primary outcome. Among others, we considered the age categories of the patients, education level, parity, previous cesarean sections, indications of cesarean section, level of the surgeon who did the $\mathrm{c} / \mathrm{s}$ however no statistically significant tendency was noticed among the infected women. The absence of any tendency might be due to the low incidence of cases during our study. Table 4 demonstrates a few of the analyzed covariates which showed a small tendency to have an impact but lacking statistical value.

Table 4 Multiple logistic regression on predictors of SSI

\begin{tabular}{llll}
\hline Regimen & OR & $\mathbf{9 5 \%} \mathbf{~ C I}$ & P-Value \\
\hline Education & 1.574 & {$[.554-4.47 I]$} & 0.395 \\
Wealth index & 5.031 & {$[.983-25.75 I]$} & 0.052 \\
Indication & 0.444 & {$[.247-.798]$} & 0.007 \\
Day or night & 0.361 & {$[.082-1.59 I]$} & 0.178 \\
Surgeon & 0.01 & {$[.272-7.233]$} & 0.066 \\
\hline
\end{tabular}

\section{Discussion}

Our study demonstrates that there is not statistically significant difference in the incidence of SSI between single dose and multiple dose of antibiotic regimens. In this study, the sample size was calculated based on the literature with a prevalence of SSI in African countries ranging between $6 \%$ to $10 \% ; ; 13,17,18$
The total number of analyzed participants was 301 women, 154 in the multiple dose group and 147 in the single dose group. Basic characteristics which could have an impact on the outcome were similar in both groups and they included age category, parity, HIV status, previous history of SSI, indication of Cesarean section, previous history of cesarean, duration of cesarean section and part of the day when the cesarean section was performed (day or night). The total rate of SSI for a period of 30 days of follow-up in our study was surprisingly small than expected as it was found to be $4.00 \%$. This low rate of SSI in our population corresponds to the findings in other studies but mainly done in middle-income countries or developed countries and not in low resource settings. ${ }^{19-22}$ The low incidence found in our study might be due to the adoption of rigorous protocols for antibiotic prophylaxis as well as hospital asepsis. A study done by Riley MMS et al on reduction of surgical site infection revealed that implementation of interventions aiming at reducing the rate of SSI reduced the cases by $63.5 \%$ in their population..$^{23}$

While the overall incidence of SSI was $4.00 \%$, the rate of SSI at discharge, which was day 3 in our study, was only $25 \%$ of all cases of SSI while the majority of new cases were seen on day 7 with a total of 8 cases corresponding to $66.6 \%$ of all cases of SSI. Only one case was discovered on day 15 and no new cases of SSI were discovered on day 30 of follow-up. This finding show that most of the cases of SSI postcesarean section develop when women are already discharged and a surveillance system or education about the signs and symptoms of SSI should be systematically done at the time of discharging women. The low rate of SSI at discharge was similarly found in a study done by Opøien HK et al, by which a tatal rate of SSI was $8,9 \%$ during an observation of 30 days post cesarean sections compared to only $1,8 \%$ observed at the time of discharge. ${ }^{24}$

Among the 147 women who received a single dose of Antibiotics, only 8 of them; $5.5 \%$ developed SSI and this incidence corresponds to the rate of SSI in other studies including one study done by Lyimo FM et al in Tanzania and a multicenter center study done in SubSharan by $\mathrm{Chu} \mathrm{K}$ et al. ${ }^{13,25}$ Interestingly, the rate of SSI among women who received multiple-dose of antibiotics for 3 days was only 4 cases; $2.6 \%$. Despite the observed difference in numbers of cases, the P value was 0.204 though not statistically significant. Based on the low occurrence of SSI in our population with an overall SSI rate of $4 \%$, a sample size of approximately 500 women would be necessary to determine the difference in both groups with a bigger strength. With the few numbers of cases of surgical site infections found in our study; both interventions are equivalent in outcome as the difference in the number of cases observed in both groups still remain in the equivalence margins and does not show any statistically significant difference to conclude otherwise. 
Some of the challenges which contributed to the limitation of our study included providers related factors; some of the healthcare providers preferred to stick on their routine practice of using extended dose of antibiotics or a packed workload prevented them from enrolling many women who were eligible to participate in the study. Another challenge in our study was related to the unexpected low prevalence of SSI, which was found in our study and leaving suspense from our conclusion. The surveillance of participants after discharge was done using phone call and this practice which is efficient for SSI may not be sensitive enough to recognize other types of infectious morbidity which may arise after $\mathrm{C} / \mathrm{S}$ though we were not able to report on secondary outcome mainly endometritis.

Our study provided information about the rate of surgical site infection in a Hospital where the timing of prophylactic antibiotics before skin incision and regular weekly operating room sterilization is respected. The overall rate of $4.00 \%$ is low compared to the general prevalence of SSI in developing countries but similar rates have been backed by other studies done in well-equipped Hospitals.

The overall observed low rate SSI in our Study might have been a result of some good practices in infections prevention practices which were systematic in Ruhengeri Hospital. These included adequate timing of prophylactic antibiotics as all women were given the first doses before they enter the operating room ensuring antibiotics are given before skin incision as recommended. The type of antibiotics was Ampicillin $2 \mathrm{~g}$ and this regimen is backed by

\section{Acknowledgments}

None.

\section{Conflicts of interest}

The authors have no conflict of interest to disclose.

\section{Funding}

This study was partially sponsored by THRiVE: The "Training Health Researchers into Vocation Excellence in East Africa".

\section{References}

1. World Health Organization. WHO Statement on Caesarean Section Rates Caesarean section rates at the hospital level and the need for a universal classification system. World Heal Organ. 2019;66(9).

2. Zejnullahu VA, Zejnullahu VA, Isjanovska R, et al. Surgical site infections after cesarean sections at the University Clinical Center of Kosovo: Rates, microbiological profile and risk factors. BMC Infect Dis. 2019;19(1):1-9.

3. Purba AKR, Setiawan D, Bathoorn E, et al. Prevention of surgical site infections: A systematic review of cost analyses in the use of prophylactic antibiotics. Frontiers in Pharmacology. 2018;9.

4. Zuarez-Easton S, Zafran N, Garmi G, et al. Postcesarean wound infection: prevalence, impact, prevention, and management challenges.

5. World Health Organization. WHO recommendations for Prevention and treatment of pre-eclampsia and eclampsia. 2011;8-27.

6. Brown J, Thompson M, Sinnya S, et al. Pre-incision antibiotic prophylaxis reduces the incidence of post-caesarean surgical site infection. $J$ Hosp Infect. 2013;83:68-70.

7. Carlet J. General principles of choice of antibiotics for antibiotic prophylaxis in surgery. Ann Fr Anesth Reanim. 1994;13(5Suppl):S10-3.
8. Ahmed ETS, Mirghani OA, Gerais AS, et al. Ceftriaxone versus ampicillin/ cloxacillin as antibiotic prophylaxis in elective caesarean section. East Mediterr Heal J. 2004;10:277-282.

9. Dahlke JD, Mendez-Figueroa H, et al. Evidence-based surgery for cesarean delivery: An updated systematic review. Am J Obstet Gynecol. 2013;209(4):294-306

10. Lamont RF, Sobel JD, Kusanovic JP, et al. Current debate on the use of antibiotic prophylaxis for caesarean section. Vol. 118, BJOG: An International Journal of Obstetrics and Gynaecology. 2011;193-201.

11. Galask RP, Benigno BB, Cunningham FG, et al. Results of a multicenter comparative study of single-dose cefotetan and multiple-dose cefoxitin as prophylaxis in patients undergoing cesarean section. American journal of surgery. 1988;155.

12. Liabsuetrakul T, Islam M. Evidence on antibiotic prophylaxis for cesarean section alone is not sufficient to change the practices of doctors in a teaching hospital. J Obstet Gynaecol Res. 2005;31:202-209.

13. Lyimo FM, Massinde AN, Kidenya BR, et al. Single dose of gentamicin in combination with metronidazole versus multiple doses for prevention of post-caesarean infection at Bugando Medical Centre in Mwanza, Tanzania: a randomized, equivalence, controlled trial. BMC Pregnancy Childbirth. 201;13(1):123.

14. Alp E, Elmali F, Ersoy S, et al. Incidence and risk factors of surgical site infection in general surgery in a developing country. Surg Today. 2014;44:685-689.

15. Ntirenganya C, Manzi O, Muvunyi CM, et al. High prevalence of antimicrobial resistance among common bacterial isolates in a tertiary healthcare facility in rwanda. Am J Trop Med Hyg. 2015;92(4):865-870.

16. Muvunyi CM, Masaisa F, Bayingana C, et al. Decreased susceptibility to commonly used antimicrobial agents in bacterial pathogens isolated from urinary tract infections in Rwanda: need for new antimicrobial guidelines. Am J Trop Med Hyg. 2011;84(6):923-928.

17. Jido T, Garba I. Surgical-site infection following cesarean section in Kano, Nigeria. Annals of Medical and Health Sciences Research. 2012;2:33.

18. Ezechi OC, Edet A, Akinlade $\mathrm{H}$, et al. Incidence and risk factors for caesarean wound infection in Lagos Nigeria. BMC Res Notes. 2009;2:186.

19. Charrier L, Serafini P, Ribatti A, et al. Post-partum surgical wound infections: Incidence after caesarean section in an Italian hospital. J Prev Med Hyg. 2009;50(3):159-163.

20. Ghuman M, Rohlandt D, Joshy G, et al. Post-caesarean section surgical site infection: rate and risk factors. $N Z$ Med J. 2011;124:32-36.

21. Habib FA. Incidence of post cesarean section wound infection in a tertiary hospital, Riyadh, Saudi Arabia. Saudi Med J. 2002;23(9):1059-1063.

22. Fan Y, Wei Z, Wang W, et al. The Incidence and Distribution of Surgical Site Infection in Mainland China: A Meta-Analysis of 84 Prospective Observational Studies. Sci Rep. 2014;4:6783.

23. Riley MMS, Suda D, Tabsh K, et al. Reduction of surgical site infections in low transverse cesarean section at a university hospital. Am J Infect Control. 2012;40(9):820-825.

24. Opøien HK, Valbø A, Grinde-et al. Post-cesarean surgical site infections according to CDC standards: rates and risk factors. A prospective cohort study. Acta Obstet Gynecol Scand. 2007;86(9):1097-1102.

25. Chu K, Maine R, Trelles M. Cesarean Section Surgical Site Infections in Sub-Saharan Africa: A Multi-Country Study from Medecins. Sans Frontieres. 2015;350-355. 